\title{
EFFECT OF DIFFERENT SOURCES AND LEVELS OF SOME DIETARY BIOLOGICAL ADDITIVES ON: II- FEED UTILIZATION BY NILE TILAPIA FISH \\ Abdelhamid', A.M.; M.E. A.Seden $^{2}$ and O.A.Zenhom ${ }^{2}$ \\ 1 Anim. Prod. Dept., Fac. of Agric., Al-Mansourah Univ., Al-Mansourah, Egypt. \\ ${ }^{2}$ Cent. Lab. For Aqua. Res., Abbasa, Abo-Hammad, Egypt.
}

\begin{abstract}
The present study aimed to investigate the effect of dietary graded levels of Aqua Superzyme, Garlen Allicin, and Diamond V (Original XPC) on feed utilization by Nile tilapia fish for 16 weeks. Dietary inclusion of the tested pre-and probiotics improved significantly $(P \leq 0.05)$ all criteria measured including feed utilization parameters, digestibility, and energy retention. The significantly $(P \leq 0.05)$ best results of feed utilization were calculated for the treatments Aqua Superzyme at $0.01 \%$, Garlen Allicin at $0.01 \%$, and Diamond V-Original XPC at $0.5 \%$ of the diet. Yet, the comparison among additives and their levels shows that the overall significantly $(\mathrm{P} \leq$ 0.05 ) best treatment was Garlen Allicin at $0.01 \%$ of the diet. Concerning the digestibility, the significantly $(P \leq 0.05)$ best level within each treatment was also Aqua Superzyme at $0.01 \%$, Garlen Allicin at $0.01 \%$, and Diamond V-Original XPC at $0.5 \%$ of the diet, respectively. The CF followed by EE were more positively affected than the other nutrients digestibility. The comparison among additives and their levels on ADC clears that the significantly $(P \leq 0.05)$ best treatment and level was Aqua Superzyme at $0.01 \%$ of the diet for ADC of all nutrients, comparing with the other additives and levels. Aqua Superzyme at $0.02 \%$, Garlen Allicin at $0.01 \%$, and Diamond V-Original $\mathrm{XPC}$ at $0.5 \%$ of the diet, respectively were the best in energy retention (in an ascending order) among their corresponding levels. The comparison among treatments revealed that best energy retention was realized by the treatment of Diamond V-Original XPC at $0.5 \%$ of the diet. Generally, it is recommended to add Garlen Allicin at $0.01 \%$ of the tilapia diet to improve its feed and nutrients utilization.

Keywords: Feed - Nutrients - Utilization - Nile tilapia.
\end{abstract}

\section{INTRODUCTION}

The research of probiotics for aquatic animals is increasing with the demand for environment friendly aquaculture. The probiotics were defined as live microbial feed supplements that biocontrol and antagonist pathogens. The most promising prospects are sketched out, but considerable efforts of research will be necessary to develop the applications to aquaculture (Castillo, 2008). Since, natural agriculture or organic farming is a return for the nature or working with, but not against, the nature (Abdelhamid, 2002). Therefore, the microbiota management tools of probiotics, prebiotics and synbiotics have been developed and, indeed, commercialized over the few decades with the expressed purpose of increasing number of bifidobacteria and/or lactobacilli within the gastrointestinal tract (Tuohy et al., 2003). So, a feeding experiment was conducted to examine the effects of dietary administration of Bacillus subtilis on feed utilization of juvenile large yellow croaker, Larimichthys crocea (mean initial body weight $7.82 \mathrm{~g} \pm 0.68$ ). The 
results showed that dietary supplementation of $1.35 \times 10^{7} \mathrm{cfu} \mathrm{g}^{-1}$ B. subtilis significantly $(P \leq 0.05)$ increased the feed efficiency ratio (FER) compared with the groups without $B$. subtilis supplementation (Ai et al., 2011). The present work aimed to study the effects of some commercial pre-and probiotics at different dietary graded levels on feed utilization by Nile tilapia fish.

\section{MATERIALS AND METHODS}

All the experimental conditions, diets, and facilities were as mentioned before in Abdelhamid et al. (2013). The $1^{\text {st }}$ and $2^{\text {nd }}$ additives used were the prebiotic Aqua Superzyme and the probiotic Garlen Allicin, each at $0.01,0.02$, and $0.03 \%$ of the diet. The $3^{\text {rd }}$ additive used was the probiotic Diamond V-Original XPC at $0.4,0.5$, and $0.6 \%$ of the diet, besides the control without additives.

Digestibility trails:

Every morning of the last two experimental weeks, feaces were collected by siphoning, separated from water, and stored at $-20{ }^{\circ} \mathrm{C}$ for analysis. Apparent digestibility coefficients (ADC) of crude protein, ether extract crude fiber, ash and nitrogen free extract were determined using the direct method according to Lovell (1989) by using the following equation:

$A D C=100$ [(nutrient intake - nutrient in feaces $) /$ nutrient intake]

Chemical analysis of diets and faeces:

The faeces were collected in a petery dish for each fish group. Uneaten feed and water were separated from the collected faeces, homogenized and stored in plastic bags at $-20^{\circ} \mathrm{C}$ for chemical analysis. The tested diets and faeces were analyzed in triplicates. Chemical analysis of feed and faeces was carried out according to the methods described by A.O.A.C. (1990) for dry matter, crude protein, ether extract, crude fiber and ash. Nitrogen free extract (carbohydrate) content was calculated by subtraction the total percentages of CP, EE, CF and ash from 100. The gross energy contents of the experimental diets and fish samples were calculated by using factors of $5.65,9.45$ and $4.2 \mathrm{Kcal} / \mathrm{g}$ of protein, lipid and carbohydrate, respectively (NRC, 1993). Digestible energy content was calculated from standard physiological fuel values as 4,4 and $9 \mathrm{Kcal} / \mathrm{g}$ of protein, carbohydrate and lipid, respectively (Garling and Wilson, 1976).

Feed utilization:

Feed conversion ration (FCR) and feed efficiency percent (FE\%) were calculated according to the following equations:

$\mathrm{FCR}=$ Feed intake $(\mathrm{g}) /$ Weight gain $(\mathrm{g})$.

$\mathrm{FE} \%=($ Weight gain $(\mathrm{g}) /$ feed intake $) \times 100$.

Protein utilization:

Protein efficiency ratio (PER) and protein productive value (PPV \%) were calculated according to the following equations:

PER = Weight gain $(\mathrm{g}) /$ Protein intake $(\mathrm{g})$.

PPV $\%=\{($ Retained protein $(\mathrm{g})) /($ protein intake $(\mathrm{g}))\} \times 100$. 
Energy utilization (NRC, 1993):

Gross energy retention (GER\%) $=\{($ Energy gain $($ Kcal $)) / G E$ intake $(\mathrm{kcal}))\} \times 100$.

Digestible energy retention $(\mathrm{DER} \%)=\{($ Energy gain $($ Kcal $)) /$ DE intake $(\mathrm{kcal}))\} \mathrm{X} 100$. Metabolizable energy retention $(M E R \%)=\{($ Energy gain $($ Kcal $)) / M E$ intake $(\mathrm{kcal}))\} \times 100$.

\section{Statistical analysis:}

Data obtained were analyzed using one-way analysis of variance which was performed according to SAS (2006). Differences were subjected to Duncan's (1955) multible range test.

\section{RESULTS AND DISCUSSION}

\section{Feed utilization:}

Mean values are given in Tables 1-3 for feed intake (FI), feed conversion ratio (FCR), feed efficiency (FE), protein efficiency ratio (FER), protein productive value (PPV), and energy utilization (EU) for Nile tilapia fish fed the experimental diets which were supplemented with the tested pre-and probiotics at their different graded levels. These Tables reflect significant $(P$ $\leq 0.05$ ) differences among levels of each additive tested for all criteria tested comparing with the control, exept EU of the $1^{\text {st }}$ two additives (Aqua Superzyme and Garlen Allicin, Tables 1 and 2, respectively). The dietary inclusion of the tested pre- and probiotics led to significantly $(P \leq 0.05)$ increased FI, FE, PER, PPV, and EU besides improving the FCR comparing with their controls. The significantly $(P \leq 0.05)$ best results were calculated for the treatments $A_{1}, G_{1}$, and $X P C_{2}$. Yet, Table 4 (comparison among additives and their levels) shows the the overall significantly $(P \leq 0.05)$ best treatment was $2^{*} 1$, i.e. $\mathrm{G}_{1}$, Garlen Allicin at $0.01 \%$ of the diet.

Table 1:Feed consumption, feed conversion ratio and nutrients utilization of Nile tilapia (O.niloticus) fed the Aqua Superzyme experimental diets.

\begin{tabular}{|l|c|c|c|c|c|c|}
\hline Treat. & FI (g) & FCR & FE (\%) & PER & PPV(\%) & EU(\%) \\
\hline Cont. & $61.04^{\mathrm{c}}$ & $2.11^{\mathrm{a}}$ & $47.41^{\mathrm{b}}$ & $1.57^{\mathrm{b}}$ & $26.89^{\mathrm{c}}$ & $20.44^{\mathrm{a}}$ \\
\hline $\mathrm{A}_{1}$ & $73.84^{\mathrm{a}}$ & $1.76^{\mathrm{b}}$ & $56.91^{\mathrm{a}}$ & $1.89^{\mathrm{a}}$ & $37.30^{\mathrm{a}}$ & $21.22^{\mathrm{a}}$ \\
\hline $\mathrm{A}_{2}$ & $66.79^{\mathrm{b}}$ & $1.86^{\mathrm{b}}$ & $53.69^{\mathrm{a}}$ & $1.78^{\mathrm{a}}$ & $34.43^{\mathrm{a}}$ & $23.08^{\mathrm{a}}$ \\
\hline $\mathrm{A}_{3}$ & $65.42^{\mathrm{b}}$ & $1.88^{\mathrm{b}}$ & $53.37^{\mathrm{a}}$ & $1.77^{\mathrm{a}}$ & $32.61^{\mathrm{a}}$ & $21.06^{\mathrm{a}}$ \\
\hline $\mathrm{P}>\mathrm{F}$ & 0.0002 & 0.0024 & 0.0021 & 0.0023 & 0.005 & 0.231 \\
\hline$\pm \mathrm{SE}$ & 1.05 & 0.042 & 1.11 & 0.037 & 1.43 & 0.85 \\
\hline
\end{tabular}

a-c: means in the same column having different letters are significantly $(P \leq 0.05)$ different. 
Table 2:Feed consumption, feed conversion ratio and nutrients utilization of Nile tilapia (O. niloticus) fed the Garlen Allicin experimental diets.

\begin{tabular}{|l|c|c|c|c|c|c|}
\hline Treat. & FI (g) & FCR & FE (\%) & PER & PPV(\%) & EU(\%) \\
\hline Cont. & $61.04^{\mathrm{d}}$ & $2.11^{\mathrm{a}}$ & $47.41^{\mathrm{c}}$ & $1.57^{\mathrm{c}}$ & $26.89^{\mathrm{c}}$ & $20.44^{\mathrm{a}}$ \\
\hline $\mathrm{G}_{1}$ & $80.04^{\mathrm{a}}$ & $1.55^{\mathrm{c}}$ & $64.46^{\mathrm{a}}$ & $2.13^{\mathrm{a}}$ & $43.26^{\mathrm{a}}$ & $24.68^{\mathrm{a}}$ \\
\hline $\mathrm{G}_{2}$ & $74.22^{\mathrm{b}}$ & $1.80^{\mathrm{b}}$ & $55.54^{\mathrm{b}}$ & $1.84^{\mathrm{b}}$ & $34.0^{\mathrm{b}}$ & $21.45^{\mathrm{a}}$ \\
\hline $\mathrm{G}_{3}$ & $69.87^{\mathrm{c}}$ & $1.93^{\mathrm{b}}$ & $51.75^{\mathrm{bc}}$ & $1.71^{\mathrm{bc}}$ & $32.33^{\mathrm{bc}}$ & $21.12^{\mathrm{a}}$ \\
\hline $\mathrm{P}>\mathrm{F}$ & 0.0001 & 0.0001 & 0.0001 & 0.0001 & 0.0016 & 0.148 \\
\hline \pm SE & 0.97 & 0.043 & 1.34 & 0.044 & 1.83 & 1.23 \\
\hline
\end{tabular}

a-d: means in the same column having different letters are significantly $(P \leq 0.05)$ different.

Table 3: Feed consumption, feed conversion ratio and nutrients utilization of Nile tilapia ( $O$. niloticus) fed the Diamond $V$ (Original XPC) experimental diets.

\begin{tabular}{|l|c|c|c|c|c|c|}
\hline Treat. & $\mathbf{F I}(\mathbf{g})$ & $\mathbf{F C R}$ & $\mathbf{F E}(\%)$ & PER & PPV(\%) & EU(\%) \\
\hline Cont. & $61.04^{\mathrm{d}}$ & $2.11^{\mathrm{a}}$ & $47.41^{\mathrm{b}}$ & $1.57^{\mathrm{b}}$ & $26.89^{\mathrm{b}}$ & $21.81^{\mathrm{b}}$ \\
\hline $\mathrm{XPC}_{1}$ & $89.95^{\mathrm{b}}$ & $1.59^{\mathrm{b}}$ & $62.69^{\mathrm{a}}$ & $2.08^{\mathrm{a}}$ & $40.25^{\mathrm{a}}$ & $24.55^{\mathrm{a}}$ \\
\hline $\mathrm{XPC}_{2}$ & $99.80^{\mathrm{a}}$ & $1.55^{\mathrm{b}}$ & $64.54^{\mathrm{a}}$ & $2.14^{\mathrm{a}}$ & $42.97^{\mathrm{a}}$ & $25.33^{\mathrm{a}}$ \\
\hline $\mathrm{XPC}_{3}$ & $83.57^{\mathrm{c}}$ & $1.58^{\mathrm{b}}$ & $63.03^{\mathrm{a}}$ & $2.09^{\mathrm{a}}$ & $39.82^{\mathrm{a}}$ & $25.08^{\mathrm{a}}$ \\
\hline $\mathrm{P}>\mathrm{F}$ & 0.0001 & 0.0001 & 0.0001 & 0.0001 & 0.0001 & 0.034 \\
\hline \pm SE & 1.31 & 0.033 & 1.02 & 0.034 & 1.183 & 0.74 \\
\hline
\end{tabular}

a-d: means in the same column having different letters are significantly $(P \leq 0.05)$ different.

Table 4:Comparison $\left(T^{*} \mathrm{~L}\right)$ among feed consumption, feed conversion ratio and nutrients utilization by Nile tilapia ( $O$. niloticus) fed Aqua Superzyme, Garlen Allicin, and Diamond V-Original XPC experimental diets.

\begin{tabular}{|l|c|c|c|c|c|c|}
\hline $\mathbf{T}^{\star} \mathbf{L}$ & FI (g) & FCR & FE (\%) & PER & PPV(\%) & EU(\%) \\
\hline Cont. & $61.04^{\mathrm{h}}$ & $2.11^{\mathrm{a}}$ & $47.41^{\mathrm{d}}$ & $1.57^{\mathrm{d}}$ & $26.89^{\mathrm{d}}$ & $20.44^{\mathrm{c}}$ \\
\hline $1^{\star} 1$ & $73.84^{\mathrm{e}}$ & $1.76^{\mathrm{c}}$ & $56.91^{\mathrm{b}}$ & $1.89^{\mathrm{b}}$ & $37.30^{\mathrm{bc}}$ & $21.22^{\mathrm{c}}$ \\
\hline $1^{\star} 2$ & $66.79^{\mathrm{fg}}$ & $1.86^{\mathrm{bc}}$ & $53.69^{\mathrm{bc}}$ & $1.78^{\mathrm{bc}}$ & $34.43^{\mathrm{c}}$ & $23.08^{\mathrm{abc}}$ \\
\hline $1^{\star} 3$ & $65.42^{\mathrm{g}}$ & $1.88^{\mathrm{bc}}$ & $53.37^{\mathrm{bc}}$ & $1.77^{\mathrm{bc}}$ & $32.61^{\mathrm{c}}$ & $21.06^{\mathrm{c}}$ \\
\hline $2^{\star} 1$ & $80.07^{\mathrm{d}}$ & $1.55^{\mathrm{d}}$ & $64.46^{\mathrm{a}}$ & $2.13^{\mathrm{a}}$ & $43.26^{\mathrm{a}}$ & $24.68^{\mathrm{ab}}$ \\
\hline $2^{\star} 2$ & $74.22^{\mathrm{e}}$ & $1.80^{\mathrm{c}}$ & $55.54^{\mathrm{b}}$ & $1.84^{\mathrm{b}}$ & $34.0^{\mathrm{c}}$ & $21.45^{\mathrm{bc}}$ \\
\hline $2^{\star} 3$ & $69.87^{\mathrm{f}}$ & $1.93^{\mathrm{b}}$ & $51.75^{\mathrm{c}}$ & $1.71^{\mathrm{c}}$ & $32.33^{\mathrm{c}}$ & $21.12^{\mathrm{c}}$ \\
\hline $3^{\star} 1$ & $89.95^{\mathrm{b}}$ & $1.59^{\mathrm{d}}$ & $62.69^{\mathrm{a}}$ & $2.08^{\mathrm{a}}$ & $40.25^{\mathrm{ab}}$ & $24.55^{\mathrm{ab}}$ \\
\hline $3^{\star} 2$ & $99.80^{\mathrm{a}}$ & $1.55^{\mathrm{d}}$ & $64.54^{\mathrm{a}}$ & $2.14^{\mathrm{a}}$ & $42.97^{\mathrm{a}}$ & $25.33^{\mathrm{a}}$ \\
\hline $3^{\star} 3$ & $83.57^{\mathrm{c}}$ & $1.58^{\mathrm{d}}$ & 63.03 & $2.09^{\mathrm{a}}$ & $39.82^{\mathrm{ab}}$ & $25.08^{\mathrm{a}}$ \\
\hline $\mathrm{P}>\mathrm{F}$ & 0.0001 & 0.0001 & 0.0001 & 0.0001 & 0.0001 & 0.0097 \\
\hline$\pm \mathrm{SE}$ & 1.17 & 0.037 & 1.182 & 0.039 & 1.60 & 1.04 \\
\hline
\end{tabular}

a-h: means in the same column having different letters are significantly $(P \leq 0.05)$ different. 
In an indoor experiment, the prebiotic Avian Plus was evaluated by adding it at graded levels into isonitrogenous-isocaloric-diets of tilapia fish for 112 days. The obtained results revealed the superiority of the Avian Plus containing diets, particularly at level of $25 \mathrm{mg} / \mathrm{kg}$ feed which led to significantly $(P \leq 0.05)$ best feed intake, feed conversion ratio (FCR), protein intake, protein productive value, and protein efficiency ratio comparing with the control (Abdelhamid et al., 2011).

Amer (2012) concluded that Diamond $V X P$ (inactive yeast a commercial product containing $100 \%$ dried Saccharomyces Cerevisae) could be used successfully as feed additive for feeding Nile tilapia Oreochromis niloticus without any adverse effects on their feed utilization. Also, results obtained showed that using Diamond $V X P$ at level $(1.5 \mathrm{~g})$ had the best results on feed utilization with levels of protein (30\%). Moreover, it could be suggested that dietary supplement with Diamond $V X P(1.5 \mathrm{~g})$ is useful in the intensive production system of fish.

Hassan (2013) and Khalil et al. (2013) obtained results concerning the effects of the probiotic Hydroyeast Aquaculture ${ }^{\circledR}$ on adult male O. niloticus showed that the $15 \mathrm{~g} / \mathrm{kg}$ diet realized best significantly $(P \leq 0.05)$ values for feed and nutrients utilization. So, based on the obtained results, the optimum level of the tested probiotic Hydroyeast Aquaculture ${ }^{\circledR}$ was depinding on fish sex.

\section{Apparent digestibility:}

The digestibility coefficients for different dietary organic nutrients were calculated at the end of the experimental period and given in Tables 5 , 6 , and 7 for the three perarations used (Aqua Superzyme, Garlen Allicin, and Diamond V-Original XPC, respectively). The ADC percentages were increased significantly $(P \leq 0.05)$ by the dietary inclusion of these tested additives comparing with the controls (without the tested additives). However, the significantly $(P \leq 0.05)$ best level within each treatment was $A_{1}, G_{1}$, and $\mathrm{XPC}_{2}$, respectively. The CF followed by $\mathrm{EE}$ were more positively affected than the other nutrients digestibility. The comparison (Table 8) among additives and their levels on ADC clears that the significantly $(P \leq 0.05)$ best treatment and level was $1 * 1$, i.e. Aqua Superzyme at $0.01 \%$ of the diet for ADC of all nutrients, comparing with the other additives and levels.

Table 5: Apparent diestibility coefficient (ADC\%) of nutrients the diets supplemented with the prebiotic (Aqua Superzyme).

\begin{tabular}{|l|c|c|c|c|c|}
\hline Treat & DM & CP & EE & CF & NFE \\
\hline Control & $85.53^{\mathrm{c}}$ & $96.52^{\mathrm{c}}$ & $76.09^{\mathrm{c}}$ & $68.60^{\mathrm{d}}$ & $81.35^{\mathrm{c}}$ \\
\hline $\mathrm{A}_{1}$ & $94.29^{\mathrm{a}}$ & $98.14^{\mathrm{a}}$ & $89.67^{\mathrm{a}}$ & $92.65^{\mathrm{a}}$ & $92.67^{\mathrm{a}}$ \\
\hline $\mathrm{A}_{2}$ & $92.09^{\mathrm{b}}$ & $97.36^{\mathrm{b}}$ & $85.30^{\mathrm{b}}$ & $87.81^{\mathrm{b}}$ & $90.56^{\mathrm{b}}$ \\
\hline $\mathrm{A}_{3}$ & $92.11^{\mathrm{b}}$ & $97.19^{\mathrm{b}}$ & $85.68^{\mathrm{b}}$ & $83.33^{\mathrm{c}}$ & $91.27^{\mathrm{b}}$ \\
\hline $\mathrm{P}>\mathrm{F}$ & 0.0001 & $0.002^{\mathrm{n}}$ & 0.0001 & 0.0001 & 0.0001 \\
\hline$\pm \mathrm{SE}$ & 0.29 & 0.18 & 0.78 & 0.69 & 0.32 \\
\hline
\end{tabular}

a-d: means in the same column having different letters are significantly $(P \leq 0.05)$ different. 
Table 6: Apparent digestibility coefficient (ADC\%) of nutrients the diets supplemented with the probiotic (Garlen Allicin).

\begin{tabular}{|l|c|c|c|c|c|}
\hline Treat & DM & CP & EE & CF & NFE \\
\hline Control & $85.53^{\mathrm{b}}$ & $96.52^{\mathrm{b}}$ & $76.09^{\mathrm{b}}$ & $68.60^{\mathrm{b}}$ & $81.35^{\mathrm{c}}$ \\
\hline $\mathrm{G}_{1}$ & $91.68^{\mathrm{a}}$ & $97.43^{\mathrm{a}}$ & $84.81^{\mathrm{a}}$ & $85.94^{\mathrm{a}}$ & $89.94^{\mathrm{ab}}$ \\
\hline $\mathrm{G}_{2}$ & $90.12^{\mathrm{a}}$ & $96.66^{\mathrm{ab}}$ & $81.05^{\mathrm{a}}$ & $81.66^{\mathrm{a}}$ & $88.48^{\mathrm{b}}$ \\
\hline $\mathrm{G}_{3}$ & $91.83^{\mathrm{a}}$ & $96.91^{\mathrm{ab}}$ & $83.11^{\mathrm{a}}$ & $83.64^{\mathrm{a}}$ & $91.01^{\mathrm{a}}$ \\
\hline $\mathrm{P}>\mathrm{F}$ & 0.0001 & 0.122 & 0.0042 & 0.0001 & 0.0001 \\
\hline \pm SE & 0.546 & 0.246 & 1.187 & 0.1471 & 0.686 \\
\hline
\end{tabular}

a-c: means in the same column having different letters are significantly $(P \leq 0.05)$ different.

Table 7:Apparent digestibility coefficient (ADC\%) of nutrients the diets supplemented with the probiotic (Diamond V-Original XPC).

\begin{tabular}{|l|c|c|c|c|c|}
\hline Treat & DM & CP & EE & CF & NFE \\
\hline Control & $85.53^{\mathrm{c}}$ & $96.52^{\mathrm{c}}$ & $76.09^{\mathrm{d}}$ & $68.60^{\mathrm{d}}$ & $81.35^{\mathrm{c}}$ \\
\hline $\mathrm{XPC}_{1}$ & $93.04^{\mathrm{a}}$ & $97.95^{\mathrm{a}}$ & $86.75^{\mathrm{b}}$ & $87.28^{\mathrm{b}}$ & $91.76^{\mathrm{a}}$ \\
\hline $\mathrm{XPC}_{2}$ & $93.85^{\mathrm{a}}$ & $98.41^{\mathrm{a}}$ & $88.98^{\mathrm{a}}$ & $92.49^{\mathrm{a}}$ & $92.33^{\mathrm{a}}$ \\
\hline $\mathrm{XPC}_{3}$ & $91.60^{\mathrm{b}}$ & $97.24^{\mathrm{b}}$ & $82.53^{\mathrm{c}}$ & $83.05^{\mathrm{c}}$ & $90.57^{\mathrm{b}}$ \\
\hline $\mathrm{P}>\mathrm{F}$ & 0.0001 & 0.0002 & 0.0001 & 0.0001 & 0.0001 \\
\hline \pm SE & 0.27 & 0.16 & 0.46 & 0.63 & 0.36 \\
\hline
\end{tabular}

a-d: means in the same column having different letters are significantly $(P \leq 0.05)$ different.

Table 8:Comparison ( $\left.T^{\star} L\right)$ among apparent diestibility coefficient (ADC\%) of nutrients of the diets supplemented with Aqua Superzyme, Garlen Allicin, and Diamond -VOriginal XPC.

\begin{tabular}{|c|c|c|c|c|c|}
\hline$T^{\star} \mathbf{L}$ & DM & CP & EE & CF & NFE \\
\hline Control & $85.53^{e}$ & $96.52^{\mathrm{e}}$ & $76.09^{9}$ & $68.60^{\mathrm{e}}$ & $81.35^{\mathrm{e}}$ \\
\hline $1 * 1$ & $94.29^{a}$ & $98.14^{a}$ & $89.67^{a}$ & $92.65^{a}$ & $92.67^{a}$ \\
\hline $1 * 2$ & $92.09^{b c}$ & $97.36^{c}$ & $85.30^{\text {cde }}$ & $87.81^{b}$ & $90.56^{b c}$ \\
\hline $1 * 3$ & $92.11^{b c}$ & $97.19^{\mathrm{cd}}$ & $85.68^{\mathrm{cd}}$ & $83.33^{\mathrm{cd}}$ & $91.27^{\mathrm{abc}}$ \\
\hline $2^{*} 1$ & $91.68^{b c}$ & $97.43^{b c}$ & $84.81^{\text {cde }}$ & $85.94^{\mathrm{bc}}$ & $89.94^{\mathrm{cd}}$ \\
\hline $2 * 2$ & $90.12^{d}$ & $96.66^{\mathrm{de}}$ & $81.05^{f}$ & $81.56^{d}$ & $88.48^{d}$ \\
\hline $2 * 3$ & $91.83^{b c}$ & $96.91^{\text {cde }}$ & $83.11^{\text {def }}$ & $83.64^{\mathrm{cd}}$ & $91.01^{\mathrm{abc}}$ \\
\hline $3^{* 1}$ & $93.04^{a b}$ & $97.95^{a b}$ & $86.75^{\mathrm{bc}}$ & $87.28^{b}$ & $91.76^{a b}$ \\
\hline $3 * 2$ & $93.85^{a}$ & $98.41^{a}$ & $88.98^{\mathrm{ab}}$ & $92.49^{a}$ & $92.33^{a}$ \\
\hline $3^{*} 3$ & $91.60^{c}$ & $97.42^{\mathrm{cd}}$ & $82.53^{\mathrm{ef}}$ & $83.05^{\mathrm{cd}}$ & $90.57^{b c}$ \\
\hline $\mathrm{P}>\mathrm{F}$ & 0.0001 & 0.0001 & 0.0001 & 0.0001 & 0.0001 \\
\hline$\pm \mathrm{SE}$ & 0.430 & 0.182 & 0.941 & 1.041 & 0.532 \\
\hline
\end{tabular}

a-g: means in the same column having different letters are significantly $(P \leq 0.05)$ fifferent.

Lara-Flores et al. (2003) evaluated the effects of probiotics on growth performance in Nile tilapia. The probiotics used based either upon Streptococcus faecium and Lactobacillus acidophilus or Saccharomyces cerevisiae. Results indicated that the fry fed diets with a probiotics supplement exhibited greater growth than those led the control diet without probiotic. Of all probiotic treatments, yeast was an appropriate growth stimulating additive in tilapia cultivation. The authors stated that the increased 
growth rate as a positive response to added probiotics could be attributed to increased efficiency of existing digestive processes or by promoting the digestion of previously indigestible substances. In addition, probiotics may have a protein sparing effect.

\section{Energy retention:}

Data of the energy retention (GER, gross energ retention; DER, digestible energy retention; and MER, metabolizable energy retention) by the tested fish fed the experimented material are given in Tables 9, 10, and 11, respectively. All forms of energy utilization were increased significantly $(P \leq$ 0.05 ) by dietary inclusion of the tested agents. $A_{2}, G_{1}$, and XPC 2 were the best (in an ascending order) among their corresponding levels. The comparison among treatments (Table 12) revealed that best energy retention was realized by the treatment of $\mathrm{XPC}_{2}$.

Table 9: Energy utilization of Nile tilapia (O. niloticus) fed the Aqua Superzyme experimental diets.

\begin{tabular}{|l|c|c|c|}
\hline Treat. & GER \% & DER \% & MER \% \\
\hline Cont. & $12.56^{\mathrm{b}}$ & $15.01^{\mathrm{b}}$ & $23.47^{\mathrm{b}}$ \\
\hline $\mathrm{A}_{1}$ & $15.53^{\mathrm{a}}$ & $18.37^{\mathrm{a}}$ & $28.49^{\mathrm{a}}$ \\
\hline $\mathrm{A}_{2}$ & $15.64^{\mathrm{a}}$ & $18.52^{\mathrm{a}}$ & $28.58 \mathrm{a}$ \\
\hline $\mathrm{A}_{3}$ & $13.88 \mathrm{a}^{\mathrm{b}}$ & $16.41 \mathrm{a}^{\mathrm{b}}$ & $25.23 \mathrm{a}^{\mathrm{b}}$ \\
\hline $\mathrm{P}>\mathrm{F}$ & 0.0286 & 0.0301 & 0.0500 \\
\hline$\pm \mathrm{SE}$ & 0.631 & 0.749 & 1.25 \\
\hline
\end{tabular}

a-b: means in the same column having different letters are significantly $(P \leq 0.05)$ different.

Table 10: Energy utilization of Nile tilapia (O. niloticus) fed the Garlen Allicin experimental diets.

\begin{tabular}{|l|c|c|c|}
\hline Treat. & GER \% & DER \% & MER \% \\
\hline Cont. & $12.56^{\mathrm{c}}$ & $15.01^{\mathrm{c}}$ & $23.47^{\mathrm{c}}$ \\
\hline $\mathrm{G}_{1}$ & $19.65^{\mathrm{a}}$ & $23.27^{\mathrm{a}}$ & $35.66^{\mathrm{a}}$ \\
\hline $\mathrm{G}_{2}$ & $15.95^{\mathrm{b}}$ & $18.85^{\mathrm{b}}$ & $29.17^{\mathrm{b}}$ \\
\hline $\mathrm{G}_{3}$ & $14.26 \mathrm{~b}^{\mathrm{c}}$ & $16.86 \mathrm{~b}^{\mathrm{c}}$ & $25.74 \mathrm{~b}^{\mathrm{c}}$ \\
\hline $\mathrm{P}>\mathrm{F}$ & 0.0005 & 0.0005 & 0.0008 \\
\hline$\pm \mathrm{SE}$ & 0.677 & 0.809 & 1.29 \\
\hline
\end{tabular}

a-c: means in the same column having different letters are significantly $(P \leq 0.05)$ different.

Table 11: Energy utilization of Nile tilapia (O. niloticus) fed the Diamond V (Original XPC) experimental diets.

\begin{tabular}{|l|c|c|c|}
\hline Treat. & GER \% & DER \% & MER \% \\
\hline Cont. & $12.56^{\mathrm{c}}$ & $15.01^{\mathrm{c}}$ & $23.47^{\mathrm{c}}$ \\
\hline $\mathrm{XPC}_{1}$ & $21.41^{\mathrm{b}}$ & $25.39^{\mathrm{b}}$ & $37.68^{\mathrm{b}}$ \\
\hline $\mathrm{XPC}_{2}$ & $24.76^{\mathrm{a}}$ & $29.42^{\mathrm{a}}$ & $45.01^{\mathrm{a}}$ \\
\hline $\mathrm{XPC}_{3}$ & $20.38^{\mathrm{b}}$ & $24.17^{\mathrm{b}}$ & $36.83^{\mathrm{b}}$ \\
\hline $\mathrm{P}>\mathrm{F}$ & 0.0001 & 0.0001 & 0.0001 \\
\hline$\pm \mathrm{SE}$ & 0.689 & 0.816 & 1.069 \\
\hline
\end{tabular}

a-c: means in the same column having different letters are significantly $(P \leq 0.05)$ different. 
Table 12: Comparison among energy utilization by Nile tilapia ( $O$. niloticus) fed the Aqua Superzyme, Garlen Allicin, and Diamond V-Original XPC experimental diets.

\begin{tabular}{|l|c|c|c|}
\hline $\mathbf{T}^{\star} \mathbf{L}$ & GER \% & DER \% & MER \% \\
\hline Cont. & $12.65^{\mathrm{d}}$ & $15.01^{\mathrm{d}}$ & $23.47^{\mathrm{e}}$ \\
\hline $1^{\star} 1$ & $15.53^{\mathrm{c}}$ & $18.37^{\mathrm{c}}$ & $28.49^{\text {cd }}$ \\
\hline $1^{\star} 2$ & $15.64^{\mathrm{c}}$ & $18.52^{\mathrm{c}}$ & $28.58^{\text {cd }}$ \\
\hline $1^{\star} 3$ & $13.88^{\mathrm{cd}}$ & $16.41^{\mathrm{cd}}$ & $25.23^{\mathrm{de}}$ \\
\hline $2^{\star} 1$ & $19.65^{\mathrm{b}}$ & $23.27^{\mathrm{b}}$ & $35.66^{\mathrm{b}}$ \\
\hline $2^{\star} 2$ & $15.95^{\mathrm{c}}$ & $18.85^{\mathrm{c}}$ & $29.17^{\mathrm{c}}$ \\
\hline $2^{\star} 3$ & $14.26^{\mathrm{cd}}$ & $16.86^{\text {cd }}$ & $25.74^{\text {cde }}$ \\
\hline $3^{\star} 1$ & $21.41^{\mathrm{b}}$ & $25.39^{\mathrm{b}}$ & $37.68^{\mathrm{b}}$ \\
\hline $3^{\star} 2$ & $24.76^{\mathrm{a}}$ & $29.42^{\mathrm{a}}$ & $45.01^{\mathrm{a}}$ \\
\hline $3^{\star} 3$ & $20.38^{\mathrm{b}}$ & $24.17^{\mathrm{b}}$ & $36.38^{\mathrm{b}}$ \\
\hline $\mathrm{P}>\mathrm{F}$ & 0.0001 & 0.0001 & 0.0001 \\
\hline$\pm \mathrm{SE}$ & 0.687 & 0.812 & 1.191 \\
\hline
\end{tabular}

a-e: means in the same column having different letters are significantly $(P \leq 0.05)$ different.

Magouz et al. (2002) concluded that adding Lacto-Sacc at $4 \mathrm{~g} / \mathrm{kg}$ diet of $O$. niloticus was the optimum for producing the best feed and protein utilization. Also, El-Ebiary and Zaki (2003) showed that feed and nutrients utilization was improved with increasing the level of active yeast up to $3 \mathrm{~g} / \mathrm{kg}$ of the diet. Since using pre-and probiotics improve the gut health (Tuohy et al., 2003). However, pro-nutrients can benefit animal health and performance through improving availability or utilization of nutrients in a variety of ways (Staykov et al., 2005). Therefore, El-Haroun et al. (2006) reported significantly $(P \leq 0.01)$ enhancement in nutrients utilization (protein efficiency ratio, protei productive value, and energy retention) by Nile tilapia fed diets included Biogen® than the control. Moreover, Yanbo and Zirong (2006) and Suzer et al. (2008) showed that probiotics highly increased the digestive enzyme activities and decreased feed conversion ratio. Additionally, ElHaroun (2007) found that Biogen ${ }^{\circledR}$ improved the feed conversion by African catfish comparing with the control. Feed conversion and protein efficiency ratio were improved too by using Biogen ${ }^{\circledR}$ in Nile tilapia diets (Mohamed et al., 2007). Bio-Mos in diets of carp, rainbow trout and European catfish decreased the feed conversion ratio (Barbu et al., 2008). 


\section{REFERENCES}

Abdelhamid, A.M. (2002). Organic Farming. Proc. $2^{\text {nd }}$ Conf. on Food borne Contamination and Egyptian's Health, April 23 - 24, Mansoura Fac. Agric., pp: 69 - 77.

Abdelhamid, A.M.; Seden, M.E.A and Zenhom, O.A. (2013). Effect of different sources and levels of some dietary biological additives on: Igrowth performance and production economy of Nile tilapia fish. J.Animal and Poultry Prod., Mansoura Univ., Vol.4 (11):615 - 635 ,2013.

Abdelhamid, A. M.; Soliman, A. A. A. and Maghraby, N. A. (2011). Evaluation of using the prebiotic avian plus in tilapia fish diet. Egyptian J. Nutrition and Feeding, 14 (3): 537-546.

Ai, Q.; Xu, H., Mai, K., Xu, W., Wang, J. and Zhang, W. (2011). Effects of dietary supplementation of Bacillus subtilis and fructooligosaccharide on growth performance, survival, non-specific immune response and disease resistance of juvenile large yellow croaker, Larimichthys crocea. Aquaculture, 317: 155-161.

Amer, A.A.A.M. (2012). Effect of different levels of protein and probiotics on productive performance of fresh water fishes. M.Sc., Fac. Agric., Kafrelsheikh Univ.

A.O.A.C. (1990): "Association of China! Agricultural Chemists" Official methods of analysis. 15 th Ed.. Published by the A.O.A.C.. Beatnin Franklin Station, Washington. D.C.

Barbu, A.; Sara, A., Alina, A. And Bentea, M. (2008). The effect of some fodder additives on production of different fish species. Buletin USAMV-CN, 65 (1-2): 1-9.

Castillo, M. (2008). Blocking pathogens with Bacillus strains. Feed-Mix, 16 (5): 19.

Duncan, D. (1955). Multiple range and multiple F-tests. Biometrics, 11: 1-42.

El-Ebiary, E.H. and Zaki, M.A. (2003). Effect of supplementing active yeast to the diets on growth performance, nutrient utilization, whole body composition and blood constituents of monosex tilapia (Oreochromis niloticus). Egypt. J. Aquat. Biol. \& Fish., 7(1): 127-139.

EL-Haroun, E.R. (2007). Improved growth rate and feed utilization in farmed African catfish Clarias gariepinus (Burchell 1822) thought a growth promoter Biogen ${ }^{\circledR}$ supplementation. Journal of Fisheries and Aquatic Science, 2: 319-327.

EL-Haroun, E.R., Goda, A. MA-S and Kabir Chowdhury, M.A. (2006). Effect of dietary probiotic Biogen ${ }^{\circledR}$ supplementation as a growth promoter on growth performance and feed utilization of Nile tilapia Oreochromis niloticus (L.). Aquaculture Research, 37: 1473-1480.

Garling, D. L. and Wilson, R. P.(1976). Optimum dietary protein to energy ratio for Channel catfish fingerlings, Ictalurus punctatus. Journal of Nutrition., (106) : 1368-1375.

Hassan, M.E.M. (2013). Physiological Studies on Fish Production. M.Sc. Thesis, Fac. Agric., Al-Mansoura Univ. 
Khalil, F.F.; Mehrim, A.I. and Hassan, M.E.M. (2013). Effect of Hydroyeast Aquaculture as growth promoter for adult Nile tilapia Oreochromis niloticus. Engormix.com, 10 p.

Lara-Flores, M., Olvera-Novoa, M.A., Guzmán-Méndez, B.E. and LópezMadrid, W. (2003). Use of the bacteria Streptococcus faecium and Lactobacillus acidophilus, and the yeast Saccharomyces cerevisiae as growth promoters in Nile tilapia Oreochromis niloticus. Aquaculture, 216: 193-201.

Lovell, R.T. (1989). Fish culture in United States. Science, 206: 1386 - 1392.

Magouz, F.I., Mohsen, M.K. and Gooda, A.H. (2002). Effect of including some biological feed additives in the diet on growth performance and feed efficiency of Nile tilapia (Oreochromis niloticus). Proc. $2^{\text {nd }}$ Conf. Foodborne Contamination and Egyptians' Health, April 23 - 24, ElMansoura Univ., pp: 329 - 339.

Mohamed, K.A., Abdel Fattah, B. and Eid, A.M.S. (2007). Evaluation of using some feed additives on growth performance and feed utilization of monosex Nile tilapia (Oreochromis niloticus) fingerlings. Agricultural Research Journal, Suez Canal University, 7: 49-54.

NRC (National Research Council) (1993). Nutrient requirements of fish. Committee on Animal Nutrition Board on Agriculture. National Academy Press, Washington DC., USA. 114pp.

SAS (2006). SAS statistical guide for personal computer, SAS Institute Inc. Cary, NC.

Staykov, Y., Spring, P. and Denev, S. (2005). Influence of dietary Bio-Mos® on growth, survival and immune status of rainbow trout (Salmo gairdneri irideus G.) and common carp (Cyprinus carpio L.). Nutrional Biotechnology in the Feed and Food Industries: Proceedings of Alltech's $21^{\text {th }}$ Annual Symposium, Nottingham University Predd, UK, pp: 333-343.

Suzer, C.; Coban, D., Kamaci, H.O., Saka, S., Firat, K., Otgucuoglu, Ö. and Küçüksari, H. (2008). Lactobacillus spp. Bacteria as probiotics in gilthead sea bream (Sparus aurata, L.) larvae: Effects on growth performance and digestive enzyme activities. Aquaculture, 280: 140145.

Tuohy, K. M., Probert, H. M., Smejkal, C. W. and Gibson, G. R. (2003). Using probiotics and prebiotics to improve gut health. DDD, 8: $692-$ 700 (www.drugdiscoverytoday.com).

Yanbo, W. and Zirong, X. (2006). Effect of probiotics for common carp (Cyprinus carpio) based on growth performance and digestive enzyme activities.Animal Feed Science and Technology, 127: 283-292. 


\section{تأثثير اختلاف مصدر ومستوى بعض الإضافات الحيوية العلفية على :}

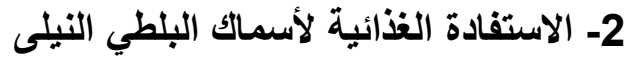

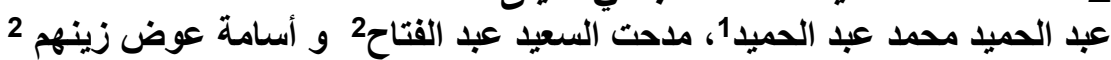

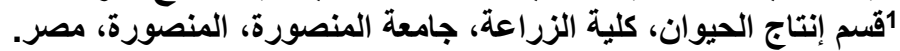

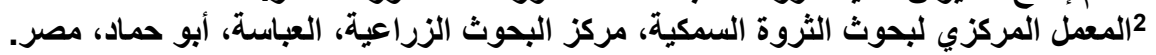

استهدفت الدراسـة الحاليـة بحث تأثير مستويات غذائيسة متدرجـة مـن كل مـن المركبـات الحيويـة

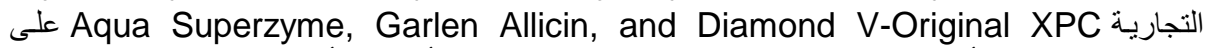

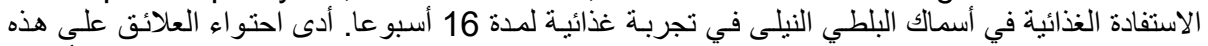

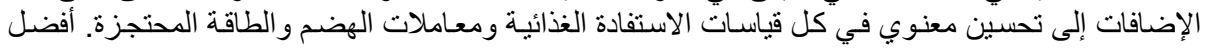

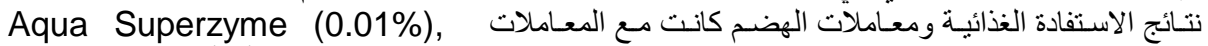
إل Garlen Allicin (0.01\%), Diamond V-Original XPC (0.5\%)

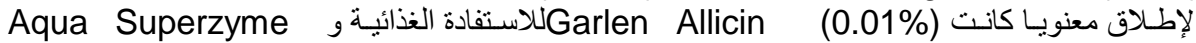

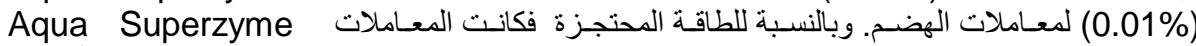
(0.02\%), Garlen Allicin (0.01\%), Diamond V-Original XPC (0.5\%)

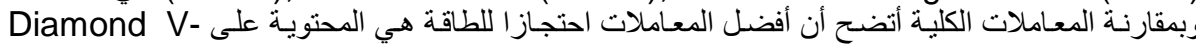
Aqua Superzyme بمستوى Original XPC بتركيز\% $0.01 \%$ من عليقة أسماك البلطي النيلى لتحسين استفادتها من الغذاء و المغذيات.

$$
\text { كلية الزراعة - جامعة المنصورة المبرة }
$$
المعمل المركزى لبحوث الثروة البمة المنمكية

\author{
قام بتحكيم البحث

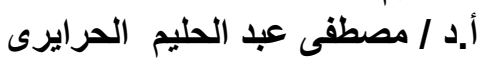 \\ أ.د / أد / محمد حسن شعبانة
}

